International Journal of Pure and Applied Mathematics

Volume 87 No. 4 2013, 535-540

ISSN: 1311-8080 (printed version); ISSN: 1314-3395 (on-line version)

url: http://www.ijpam.eu

doi: http://dx.doi.org/10.12732/ijpam.v87i4.4

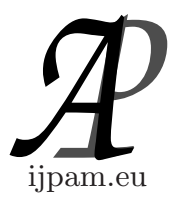

\title{
A BOUND ON THE POISSON BINOMIAL-POISSON RELATIVE ERROR
}

\author{
K. Teerapabolarn \\ Department of Mathematics \\ Faculty of Science \\ Burapha University \\ Chonburi, 20131, THAILAND
}

\begin{abstract}
We use the Stein-Chen method to determine a bound on the relative error between the Poisson binomial distribution function with parameter $\mathbf{p}=\left(p_{1}, \ldots, p_{n}\right)$ and the Poisson distribution function with mean $\lambda=\sum_{i=1}^{n} \frac{p_{i}}{1-p_{i}}$. With this bound, the Poisson distribution function with this mean can be used as an estimate of the Poisson binomial distribution function whenever all $p_{i}$ are small.
\end{abstract}

AMS Subject Classification: 62E17, 60F05, 60G50

Key Words: distribution function, Poisson distribution, Poisson binomial distribution, relative error, Stein-Chen method

\section{Introduction}

Let $Y_{1}, \ldots, Y_{n}$ be $n$ independently distributed Bernoulli random variables, each with probability of success $p_{i}=P\left(Y_{i}=1\right)=1-P\left(Y_{i}=0\right)$, and let $X=$ $\sum_{i=1}^{n} Y_{i}$. Then the distribution of $X$ is called the Poisson binomial distribution with parameter $\mathbf{p}=\left(p_{1}, \ldots, p_{n}\right)$. This distribution can be thought of as the distribution of the number of successes in a sequence of $n$ independent Bernoulli trials, where success occurs on each $i$ th trial with a probability of $p_{i}$ and failure occurs on each $i$ th trial with a probability of $q_{i}=1-p_{i}$. The probability function of $X[4]$ is of the form

Received: May 7, 2013

(c) 2013 Academic Publications, Ltd. url: www.acadpubl.eu 


$$
P(X=k)=\sum_{\mathbf{d} \in D^{k}} \prod_{i=1}^{n}\left(\frac{p_{i}}{q_{i}}\right)^{d_{i}} \prod_{j=1}^{n} q_{j}, k=0,1, \ldots, n,
$$

where $D^{k}=\left\{\mathbf{d}=\left(d_{1}, \ldots, d_{n}\right): d_{i}=0,1 ; d_{1}+\cdots+d_{n}=k\right\}$, and the mean and variance of $X$ are $E(X)=\sum_{i=1}^{n} p_{i}$ and $\operatorname{Var}(X)=\sum_{i=1}^{n} p_{i} q_{i}$, respectively. It is well known that the Poisson distribution with mean $\sum_{i=1}^{n} p_{i}$ can be used as an estimate of the Poisson binomial distribution with parameter $\mathbf{p}$ if $n$ is large and all $p_{i}$ are small. In the past few years, many authors have tried to approximate the Poisson binomial distribution by the Poisson distribution with mean $\lambda=\sum_{i=1}^{n} p_{i}$. For example, Barbour et al. [1] gave a uniform bound for the difference of the Poisson binomial and Poisson distributions

$$
\left|\sum_{k \in A} \sum_{\mathbf{d} \in D^{k}} \prod_{i=1}^{n}\left(\frac{p_{i}}{q_{i}}\right)^{d_{i}} \prod_{j=1}^{n} q_{j}-\sum_{k \in A} \frac{e^{-\lambda} \lambda^{k}}{k !}\right| \leq \lambda^{-1}\left(1-e^{-\lambda}\right) \sum_{i=1}^{n} p_{i}^{2},
$$

where $A \subseteq \mathbb{N} \cup\{0\}$. In the case of pointwise approximation, Neammanee [5] gave a non-uniform bound for the difference of the Poisson binomial and Poisson probability functions

$$
\left|\sum_{\mathbf{d} \in D^{x_{0}}} \prod_{i=1}^{n}\left(\frac{p_{i}}{q_{i}}\right)^{d_{i}} \prod_{j=1}^{n} q_{j}-\frac{e^{-\lambda} \lambda^{x_{0}}}{x_{0} !}\right| \leq \min \left\{\frac{1}{x_{0}}, \lambda^{-1}\right\} \sum_{i=1}^{n} p_{i}^{2},
$$

where $x_{0} \in\{1, \ldots, n-1\}$. For cumulative probability approximation, the Poisson binomial distribution function

$$
\mathbb{P B}_{\mathbf{p}}(k)=\sum_{j=0}^{k} \sum_{\mathbf{d} \in D^{j}} \prod_{i=1}^{n}\left(\frac{p_{i}}{q_{i}}\right)^{d_{i}} \prod_{j=1}^{n} q_{j}, k=0,1, \ldots, n
$$

is also approximated by the Poisson distribution function

$$
\mathbb{P}_{\lambda}(k)=\sum_{j=0}^{k} \frac{e^{-\lambda} \lambda^{j}}{j !}, k=0,1, \ldots,
$$

for $\lambda=\sum_{i=1}^{n} p_{i}$. In this case, Teerapabolarn and Neammanee [7] gave a nonuniform bound in the form of

$$
\left|\mathbb{P B}_{\mathbf{p}}\left(x_{0}\right)-\mathbb{P}_{\lambda}\left(x_{0}\right)\right| \leq \lambda^{-1}\left(1-e^{-\lambda}\right) \min \left\{1, \frac{e^{\lambda}}{x_{0}+1}\right\} \sum_{i=1}^{n} p_{i}^{2}
$$


where $x_{0} \in\{0,1, \ldots, n\}$. Note that, the results in (1.2), (1.3) and (1.6) were created by the Stein-Chen method.

Following the Law of Small Numbers, it is observed that the Poisson distribution with mean $\lambda=\sum_{i=1}^{n} \frac{p_{i}}{q_{i}}$ can also be used as an estimate of the Poisson binomial distribution with parameter $\mathbf{p}$ when $n$ is large and all $p_{i}$ are small. Similarly, the Poisson binomial cumulative distribution function can also be properly approximated by the Poisson cumulative distribution function with mean $\lambda=\sum_{i=1}^{n} \frac{p_{i}}{q_{i}}$. Let $\mathbb{P B}_{\mathbf{p}}\left(x_{0}\right)$ and $\mathbb{P}_{\lambda}\left(x_{0}\right)$ be the Poisson binomial and Poisson distribution functions at $x_{0} \in\{0,1, \ldots, n\}$. In this paper, we are interested to approximate $\mathbb{P B}_{\mathbf{p}}\left(x_{0}\right)$ by $\mathbb{P}_{\lambda}\left(x_{0}\right)$ with mean $\lambda=\sum_{i=1}^{n} \frac{p_{i}}{q_{i}}$ in terms of the relative error $1-\frac{\mathbb{P}_{\lambda}\left(x_{0}\right)}{\mathbb{P B}_{\mathbf{p}}\left(x_{0}\right)}$ and its bound.

The Stein-Chen method is the tool for deriving a bound for the such relative error, which is mentioned in Section 2. In Section 3, the Stein-Chen method is applied to obtain the desired result. Conclusion is presented in the last section.

\section{Method}

Stein's method was originally formulated for normal approximation [3]. It was adapted and applied to the Poisson case by Chen [2], which is refer to as the Stein-Chen method. Following Teerapabolarn [6], Stein's equation of the Poisson distribution function with parameter $\lambda>0$ is of the form

$$
h_{x_{0}}(x)-\mathbb{P}_{\lambda}\left(x_{0}\right)=\lambda f_{x_{0}}(x+1)-x f_{x_{0}}(x),
$$

where $x_{0}, x \in \mathbb{N} \cup\{0\}$, and for $h_{x_{0}}(x)=1$ if $x \leq x_{0}$ and $h_{x_{0}}(x)=0$ if $x>x_{0}$. For $x_{0} \in \mathbb{N}$, he showed that

$$
0<\sup _{x \geq 2} f_{x_{0}}(x) \leq \frac{\lambda^{-2}\left(e^{\lambda}-\lambda-1\right) \mathbb{P}_{\lambda}\left(x_{0}\right)}{x_{0}+1} .
$$

\section{Result}

The following theorem shows a result of the Poisson approximation to the Poisson binomial distribution function in the relative error form and its bound. 
Theorem 3.1. For $x_{0} \in\{1, \ldots, n\}$ and $\lambda=\sum_{i=1}^{n} \frac{p_{i}}{q_{i}}$, then the following inequality holds:

$$
0 \leq 1-\frac{\mathbb{P}_{\lambda}\left(x_{0}\right)}{\mathbb{P B}_{\mathbf{p}}\left(x_{0}\right)} \leq \frac{\lambda^{-2}\left(e^{\lambda}-\lambda-1\right)}{x_{0}+1} \sum_{i=1}^{n} \frac{p_{i}^{2}}{q_{i}}
$$

where $1-\frac{\mathbb{P}_{\lambda}\left(x_{0}\right)}{\mathbb{P B}_{p}\left(x_{0}\right)}=1-\frac{e^{-\lambda}}{\prod_{i=1}^{n} q_{i}}$ when $x_{0}=0$.

Proof. Substituting $x$ by $X$ and taking expectation in (2.1), yields

$$
\begin{aligned}
\mathbb{P B}_{\mathbf{p}}\left(x_{0}\right)-\mathbb{P}_{\lambda}\left(x_{0}\right) & =E[\lambda f(X+1)-X f(X)] \\
& =E\left[\sum_{i=1}^{n} \frac{p_{i}}{q_{i}} f(X+1)-\sum_{i=1}^{n} Y_{i} f(X)\right] \\
& =\sum_{i=1}^{n} E\left[\frac{p_{i}}{q_{i}} f(X+1)-Y_{i} f(X)\right],
\end{aligned}
$$

where $f=f_{x_{0}}$ is defined as in (2.1). Let $\delta(f)=E\left[\frac{p_{i}}{q_{i}} f(X+1)-Y_{i} f(X)\right]$ and let $X_{i}=X-Y_{i}$. Then, for each $i$,

$$
\begin{aligned}
\delta(f)= & E\left[\frac{p_{i}}{q_{i}} f\left(X_{i}+Y_{i}+1\right)-Y_{i} f\left(X_{i}+Y_{i}\right)\right] \\
= & E\left\{E\left[\left(\frac{p_{i}}{q_{i}} f\left(X_{i}+Y_{i}+1\right)-Y_{i} f\left(X_{i}+Y_{i}\right)\right) \mid Y_{i}\right]\right\} \\
= & E\left[\left(\frac{p_{i}}{q_{i}} f\left(X_{i}+Y_{i}+1\right)-Y_{i} f\left(X_{i}+Y_{i}\right)\right) \mid Y_{i}=0\right] q_{i} \\
& +E\left[\left(\frac{p_{i}}{q_{i}} f\left(X_{i}+Y_{i}+1\right)-Y_{i} f\left(X_{i}+Y_{i}\right)\right) \mid Y_{i}=1\right] p_{i} \\
= & E\left[p_{i} f\left(X_{i}+1\right)\right]+E\left[\frac{p_{i}^{2}}{q_{i}} f\left(X_{i}+2\right)-p_{i} f\left(X_{i}+1\right)\right] \\
= & \frac{p_{i}^{2}}{q_{i}} E\left[f\left(X_{i}+2\right)\right] .
\end{aligned}
$$

Combining (3.2) and (3.3) and using (2.2), it follows that

$$
\begin{aligned}
0 \leq \mathbb{P B}_{\mathbf{p}}\left(x_{0}\right)-\mathbb{P}_{\lambda}\left(x_{0}\right) & =\sum_{i=1}^{n} \frac{p_{i}^{2}}{q_{i}} E\left[f\left(X_{i}+2\right)\right] \\
& \leq \sum_{i=1}^{n} \frac{p_{i}^{2}}{q_{i}} \sup _{x \geq 2} f(x)
\end{aligned}
$$




$$
\leq \frac{\lambda^{-2}\left(e^{\lambda}-\lambda-1\right) \mathbb{P}_{\lambda}\left(x_{0}\right)}{x_{0}+1} \sum_{i=1}^{n} \frac{p_{i}^{2}}{q_{i}} .
$$

Dividing (3.4) by $\mathbb{P B}_{\mathbf{p}}\left(x_{0}\right)$ and since $\frac{\mathbb{P}_{\lambda}\left(x_{0}\right)}{\mathbb{P B}_{\mathbf{p}}\left(x_{0}\right)} \leq 1$, it follows that the inequality (3.1) holds.

Remark. Consider the result in Theorem 3.1, if all $p_{i}$ are small, then the bound (3.1) approaches 0 , that is, the Poisson binomial distribution function can be properly approximated by the Poisson distribution with this mean.

\section{Conclusion}

A bound in Theorem 3.1 is an estimate of the relative error between the Poisson binomial distribution function with parameter $\mathbf{p}=\left(p_{1}, \ldots, p_{n}\right)$ and the Poisson distribution with mean $\lambda=\sum_{i=1}^{n} \frac{p_{i}}{q_{i}}=\sum_{i=1}^{n} \frac{p_{i}}{1-p_{i}}$. It is indicated that the Poisson distribution function with this mean can be used as an estimate of the Poisson binomial distribution function whenever all $p_{i}$ are small.

\section{References}

[1] A.D. Barbour, L. Holst, S. Janson, Poisson Approximation, Oxford Studies in Probability 2, Clarendon Press, Oxford, 1992.

[2] L.H.Y. Chen, Poisson approximation for dependent trials, Ann. Probab., 3 (1975), 534-545.

[3] C.M. Stein, A bound for the error in normal approximation to the distribution of a sum of dependent random variables, Proc. Sixth Berkeley Sympos. Math. Statist. Probab., 3 (1972), 583-602.

[4] S.X. Chen, J.S. Liu, Statistical applications of the Poisson-binomial and condition bernoulli distributions. Statist. Sinica, 7 (1997), 875-892.

[5] K. Neammanee, Pointwise Approximation of Poisson binomial by Poisson distribution. Stochastic Modelling and Applications, 6 (2003), 20-26.

[6] K. Teerapabolarn, A new bound on Poisson approximation for independent geometric variables, International Journal of Pure and Applied Mathematics, 84 (2013), 419-422. 
[7] K. Teerapabolarn, K. Neammanee, Poisson approximation for sums of dependent Bernoulli random variables. ActaMathematica Academiae Paedagogicae Nyiregyhaziensis, 22 (2006), 87-99. 